\title{
Model Spectra for Accretion Disks Truncated at the Inner Edge
}

\section{RICHARD A. WADE, ${ }^{1}$ MARCOS DIAZ, ${ }^{1}$ AND IVAN HUBENY ${ }^{2}$}

\author{
${ }^{1}$ Department of Astronomy \& Astrophysics, 525 Davey Lab, \\ Pennsylvania State University, University Park, PA 16802 \\ ${ }^{2}$ Goddard Space Flight Center, Code 681, Greenbelt, MD 20771
}

Attempts to model the FUV spectra of accretion disks in cataclysmic variables have suggested that in some cases the innermost part of the disk is "missing". We present computed spectra of accretion disks with various amounts of the inner disk removed arbitrarily, to illustrate whether the absence of the inner disk can be detected via the profiles of strong lines. Results are presented for lines in the UV and FUV for selected disk parameters and inclinations. UV/FUV line diagnostics can in principle establish whether the hot inner disk is present as expected from standard steady-state disk theory. The presence or absence of the inner disk has direct impact on the interpretation of the EUV spectra of cataclysmic variables, in particular the boundary layer emission.

\section{Introduction}

The visible, UV/FUV, and EUV light from a cataclysmic variable binary (CV) arises from an accretion structure which is inherently multi-temperature. The EUV spectrum of an accretion disk around a white dwarf is expected to be dominated by emission from the boundary layer (BL), rather than from the disk proper, but analysis of several systems in recent years has suggested that the BL luminosity is less than predicted. To make progress on this question, an accurate model of the disk emission in the EUV, based on UV/FUV and visible light data (where the BL contribution should be much less), is needed. But how well can we model the UV/FUV emission from CVs?

\section{IX Velorum}

Long, et al. (1993) discussed the FUV spectrum of the novalike variable IX Velorum, observed by the Hopkins Ultraviolet Telescope (HUT) during the ASTRO-1 mission. IX Vel contains a cool secondary star, which feeds an accretion disk around an $0.8 \mathrm{M}_{\odot}$ white dwarf. The disk is viewed at an angle 60 degrees from face-on. IX Vel is about 95 pc distant. (Data from Beuermann \& Thomas 1990.)

The IX Vel spectrum showed a strong, blue continuum upon which was superposed a pattern of resonance lines ( $P$ Cygni profiles or blue shifted absorption profiles) from the wind. The Lyman series of hydrogen and some other features were present in absorption, kinematically blended due to the Keplerian orbital motions of gas in the disk.

Attempts to model the FUV spectrum of IX Vel suggested that the innermost part of the disk is "missing". Steady-state models of the disk, assumed to extend inward to the white dwarf surface, and with the mass transfer rate $\dot{M}$ chosen to match the overall observed UV flux, did not match the shape of the observed spectrum particularly well. An improvement was possible, however, if the inner portion of the disk was "removed" from the model flux calculation, with a compensating adjustment of $\dot{M}$ to maintain the observed overall flux level. The hottest regions of the disk were removed by this treatment, resulting in a change in the shape of the disk spectrum, especially near the Lyman limit. 


\section{Line Diagnostics of a Missing Inner Disk}

Based only on the continuum shape, a conclusion that the inner disk is missing cannot be considered to be firmly established. Does interpretation of line profiles support this conclusion? The presence of a strong wind in IX Vel makes use of lines difficult for that system, but other $\mathrm{CVs}$, observed at high $\mathrm{S} / \mathrm{N}$ and adequate spectral dispersion, might prove amenable to analysis. We present some illustrative line profile calculations, to explore whether non-standard disk structures can be detected via line profiles.

In a standard steady-state disk, the effective temperature and orbital velocity of the disk vary with radial distance from the central white dwarf, with the inner disk being the hottest region:

$$
T_{e f f}(r) \propto r^{-3 / 4} \times\left[1-\left(r_{w d} / r\right)^{1 / 2}\right]^{1 / 4}
$$

The Doppler shifts will be $v_{p r o j}(r, \theta) \propto r^{-1 / 2} \sin i \cos \theta$, where $\theta$ is an azimuthal angle in the disk. The use of kinematic broadening of lines that form at different characteristic temperatures allows one in principle to detect whether the inner disk is present. This hope might be frustrated by too severe blending of lines, or by the formation of lines over sufficiently large ranges of disk radius that the line profile "tool" is effectively blunted.

\section{Choice of Models}

We adopt a white dwarf mass of $0.9 \mathrm{M}_{\odot}$ and radius of $r_{w d}=6.2 \times 10^{8} \mathrm{~cm}$. We consider two accretion rates, $\dot{M}=2 \times 10^{-9}$ and $1 \times 10^{-8} \mathrm{M}_{\odot} \mathrm{yr}^{-1}$. We construct models of the whole disk, or of "evacuated" disks, where $r_{i n}>r_{w d}$. The disk is placed at $100 \mathrm{pc}$, and viewed from several inclination angles, $i$.

Note that we make an ad hoc truncation of the inner disk, without considering changes in the remaining disk structure $T(r)$. This is not entirely self-consistent, but is justified in a preliminary exploration.

The local vertical structure of the disk at each radius is computed using the code TLUSDISK (Hubeny 1988, 1991), and the emerging spectrum (flux and specific intensity) is calculated using the codes SYNSPEC (Hubeny, Lanz \& Jeffery 1994: rest frame) and DISKSYN (Keplerian motion and integration over the disk surface).

\section{Results}

The figures show sample calculated disk spectra in the regions near C IV 1548,1551 $\AA$ and the H Lyman lines. Each region contains one or more features that are formed chiefly in the outer disk, which are little altered by the removal of the inner disk and can be used as a reference for comparison.

Each sample is shown at two inclinations, to illustrate the strong effect of viewing angle. Each panel shows a single $\dot{M}$ with various amounts of inner disk removed $\left(r_{i n}\right.$ parameter).

The C IV $1550 \AA$ doublet in Figure 1 is formed mainly at $r<3 r_{w d}$, and the equivalent width of the line is reduced nearly to zero as $r_{i n}$ increases. At the higher $\dot{M}$ of the models in Table 2, the same is true for the He II line at $1640 \AA$ (not shown), for which useful "outer disk" lines are Fe II 1611, $1612 \AA$, C I $1657 \AA$, and Al II $1671 \AA$. Both the C IV and He II lines may be strongly affected by a wind from the disk, however.

The Lyman line region (Figure 2) contains features near $1085 \AA(\mathrm{He}$ II $+\mathrm{N} \mathrm{II}$ ) and $1066 \AA$ (C II) whose ratio varies by a factor of $2-3$ as $r_{i n}$ changes in the higher $\dot{M}$ model. This region may be free of excessive contamination by wind lines. The profiles of the Lyman lines themselves (blended with He II) also vary significantly. However, Long et al. 


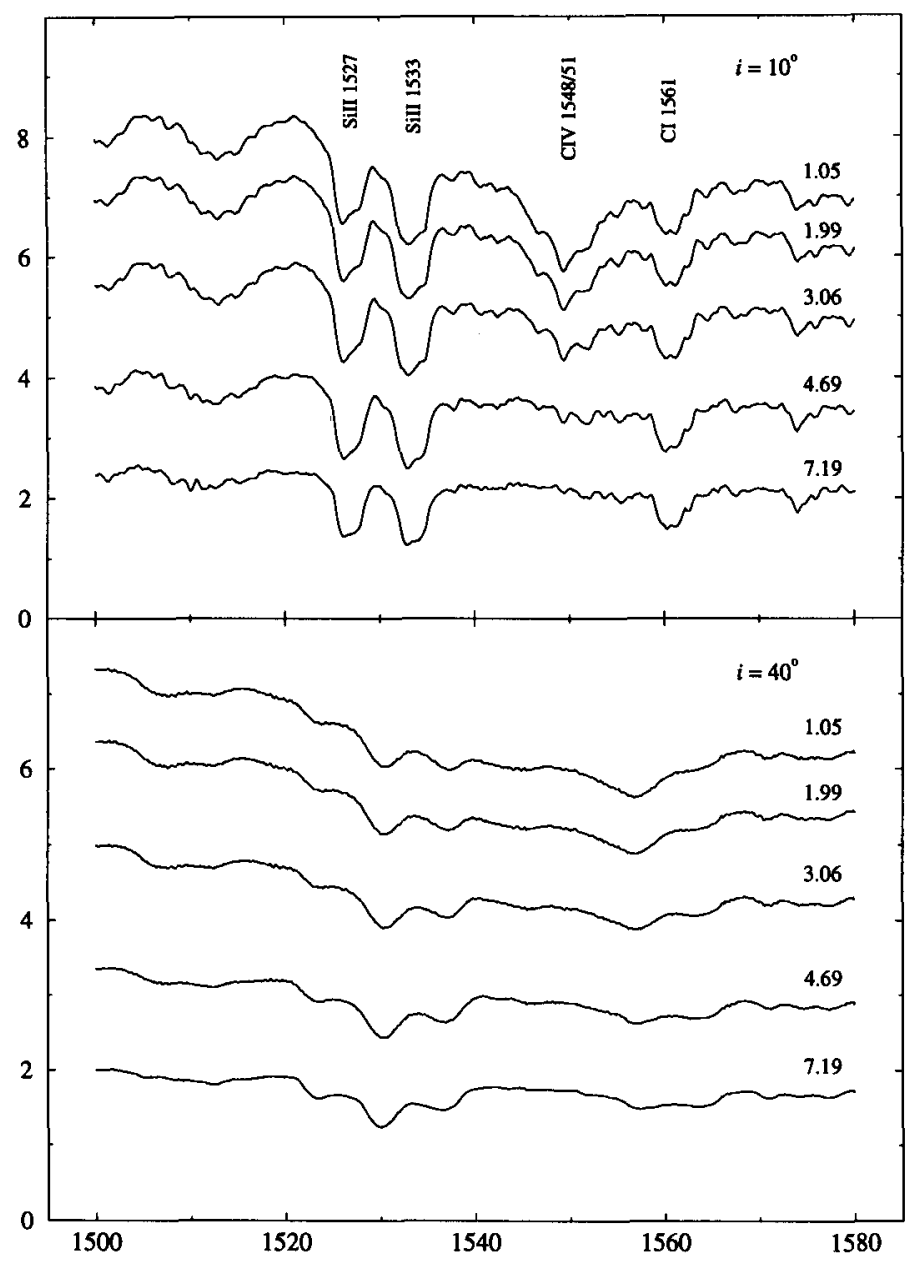

Figure 1. The region near C IV for the models specified in Table 1. The upper panel shows spectra for a disk inclination of $i=10^{\circ}$, and the lower panel shows $i=40^{\circ}$. Numbers at the right end of each spectrum specify the ratio $r_{i n} / r_{w d}$. Wavelengths are in $\AA$ units. Flux is given in units of $10^{-12} \mathrm{erg} \mathrm{cm} \mathrm{cm}^{-2} \mathrm{~s}^{-1} \AA^{-1}$ for a distance of $100 \mathrm{pc}$, with no geometric foreshortening taken into account. 


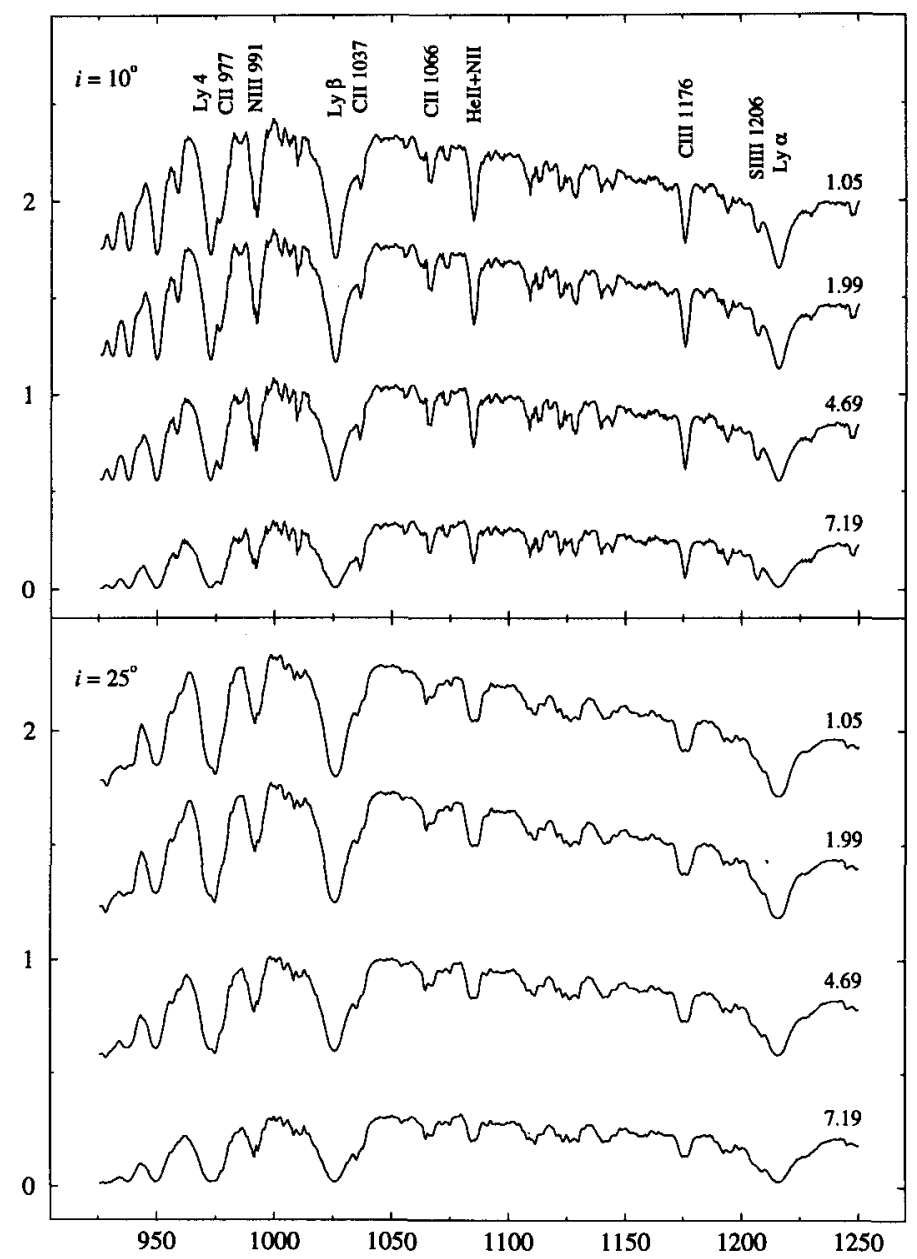

Figure 2. The H Lyman line region for the models specified in Table 2. The upper panel shows spectra for a disk inclination of $i=10^{\circ}$, and the lower panel shows $i=25^{\circ}$. Wavelengths are in $\AA$ units. Flux is given in units of $10^{-10} \mathrm{erg} \mathrm{cm}{ }^{-2} \mathrm{~s}^{-1} \AA^{-1}$ for a distance of $100 \mathrm{pc}$, with no geometric foreshortening taken into account. Successively higher spectra in each panel are displaced upwards by 0.5 units to avoid overlap. 
TABLE 1. Model details for $M=0.9 \mathrm{M}_{\odot}, \dot{M}=2 \times 10^{-9} \mathrm{M}_{\odot} \mathrm{yr}^{-1}$

$\begin{array}{lcrr}r_{i n} / r_{w d} & T\left(r_{i n}\right) & v\left(r_{i n}\right) i=10^{\circ} & v\left(r_{i n}\right) i=40^{\circ} \\ 1.05 & 40,800 \mathrm{~K} & 740 \mathrm{~km} \mathrm{~s}^{-1} & 2750 \mathrm{~km} \mathrm{~s}^{-1} \\ 1.99 & 47,000 \mathrm{~K} & 540 \mathrm{~km} \mathrm{~s}^{-1} & 2000 \mathrm{~km} \mathrm{~s}^{-1} \\ 3.06 & 37,500 \mathrm{~K} & 440 \mathrm{~km} \mathrm{~s}^{-1} & 1610 \mathrm{~km} \mathrm{~s}^{-1} \\ 4.69 & 28,800 \mathrm{~K} & 350 \mathrm{~km} \mathrm{~s}^{-1} & 1300 \mathrm{~km} \mathrm{~s}^{-1} \\ 7.19 & 21,700 \mathrm{~K} & 280 \mathrm{~km} \mathrm{~s}^{-1} & 1050 \mathrm{~km} \mathrm{~s}^{-1}\end{array}$

TABLE 2. Model details for $M=0.9 \mathrm{M}_{\odot}, \dot{M}=10^{-8} \mathrm{M}_{\odot} \mathrm{yr}^{-1}$

$\begin{array}{lcrr}r_{i n} / r_{w d} & T\left(r_{i n}\right) & v\left(r_{i n}\right) i=10^{\circ} & v\left(r_{i n}\right) i=25^{\circ} \\ 1.05 & 61,000 \mathrm{~K} & 740 \mathrm{~km} \mathrm{~s}^{-1} & 1800 \mathrm{~km} \mathrm{~s}^{-1} \\ 1.99 & 70,300 \mathrm{~K} & 540 \mathrm{~km} \mathrm{~s}^{-1} & 1320 \mathrm{~km} \mathrm{~s}^{-1} \\ 4.69 & 43,200 \mathrm{~K} & 350 \mathrm{~km} \mathrm{~s}^{-1} & 860 \mathrm{~km} \mathrm{~s}^{-1} \\ 7.19 & 32,500 \mathrm{~K} & 280 \mathrm{~km} \mathrm{~s}^{-1} & 690 \mathrm{~km} \mathrm{~s}^{-1}\end{array}$

suggested that blends with wind lines (e.g., O VI, N IV, S VI) or geocoronal lines (Ly $\alpha$ ) limit their individual usefulness.

We conclude that for some disk inclinations and mass transfer rates, a combination of line shape and equivalent width for lines formed at different characteristic temperatures may be useful in assessing whether the disk is truncated from the inside. A complete modeling of the UV/FUV spectrum must of course take account of the shape and level of the continuum as well. If the disk is truncated, the predicted EUV spectrum will be severely altered from the standard (intact disk) model.

The equivalent width diagnostic becomes more difficult but not impossible to use at higher inclination, due to severe blending and spreading of the line profiles. Accurate photometric calibration and adequate signal to noise are essential.

Supported by NASA grant NAGW-3171.

\section{REFERENCES}

Beuermann, K. \& Thomas, H. -C. 1990, Detection of emission lines from the secondary star in IX Velorum $=\mathrm{CPD}-48^{\circ} 1577$, A\&A, 230,326

HuBENY, I. 1988, A computer program for calculating non-LTE model stellar atmospheres, Comp. Phys. Comm., 52, 103

Hubeny, I. 1991, Model atmospheres for accretion disks, In Structure and Emission Properties of Accretion Disks, ed. C. Bertont et al., IAU Colloquium, Editions Frontières, 129, 227,

HubENy, I., LANZ, T., \& JeFFERY, C. S. 1994, TLUSTY \& SYNSPEC-a user's guide, Collaborative Computational Project 7, documentation file

Long, K. S., Wade, R. A., Blair, W. P., Davmsen, A. F., \& Hubeny, I. 1993, Observations of the bright nova-like variable IX Velorum with the Hopkins Ultraviolet Telescope., ApJ, 426,704 